\title{
Maisons en terre archéologique à Mahasthangarh (Bangladesh)
}

Archaeological earth houses in Mahasthangarh (Bangladesh)

Casas en «tierra arquelógica » de Mahasthangarh (Bangladesh)

\section{Anie Montigny et Séverine Sanz}

\section{OpenEdition}

\section{Journals}

Édition électronique

URL : https://journals.openedition.org/tc/62

DOI : $10.4000 /$ tc. 62

ISSN : 1952-420X

Éditeur

Éditions de l'EHESS

Édition imprimée

Date de publication : 1 décembre 2003

Pagination : 65-89

ISSN : 0248-6016

Référence électronique

Anie Montigny et Séverine Sanz, « Maisons en terre archéologique à Mahasthangarh (Bangladesh) », Techniques \& Culture [En ligne], 41 | 2003, mis en ligne le 13 janvier 2005, consulté le 29 septembre 2022. URL : http://journals.openedition.org/tc/62 ; DOI : https://doi.org/10.4000/tc.62

Ce document a été généré automatiquement le 29 septembre 2022.

Tous droits réservés 


\title{
Maisons en terre archéologique à Mahasthangarh (Bangladesh)
}

\author{
Archaeological earth houses in Mahasthangarh (Bangladesh) \\ Casas en « tierra arquelógica » de Mahasthangarh (Bangladesh)
}

Anie Montigny et Séverine Sanz

1 Les maisons de Mahasthangarh sont bien des maisons en terre -en terre crue- et non des maisons à base de terre comme certaines autres dans les villages environnants. Problème de définition : K. I. Ahmed (1994:2) se pose la question en faisant référence à l'architecture rurale du Bangladesh, qui est diversifiée (bambou, terre, clayonnage avec torchis). C'est pourquoi il choisit le second terme ${ }^{3}$.

2 La particularité de Mahasthangarh est qu'il s'agit d'un vaste site archéologique sur lequel vivent les habitants ${ }^{4}$. La terre de construction des maisons est donc réemployée, pourvue de débris de poterie et de nodules de briques. Une terre excellente aux dires des villageois.

3 Mais c'est bien un habitat de pauvres, si l'on en juge par l'espoir des habitants de faire construire en dur s'ils en avaient les moyens. Ce type d'habitat est exclusivement rural.

\section{Un emplacement privilégié : le site archéologique}

4 Mahasthangarh est ce qu'on appelle au Proche-Orient, un tell. C'est une butte formée par l'accumulation des couches anthropiques, alternant constructions et destructions de bâtiments en terre. Le site apparaît très nettement surélevé par rapport à la plaine d'inondation de la rivière Karatoya qui longe la citadelle, à l'est. Lors de la fouille du "rempart est», le sondage profond a révélé que les couches archéologiques mesuraient, à cet endroit, 5 à $6 \mathrm{~m}$ d'épaisseur : le sol actuel était situé à $23 \mathrm{~m}$, et le sol vierge à une altitude absolue de 17,50 m (Bernard, Boussac, Breuil \& Salles 2001), la route Mahasthangarh/Shibganj, longeant le site en contrebas se situe -juste hors inondation en période de mousson- à une altitude de $16 \mathrm{~m}$. Il semble donc que la présence d'une éminence topographique ait préexisté au tell. Nous sommes en effet à 
l'exacte limite des terrasses du Barind, unité géomorphologique datant du Pléistocène, formée par le dépôt sédimentaire d'un ancien système hydrographique associé à la rivière Tista (Jacqueminet, Allemand \& Pedoja 2001), dont le cours est désormais totalement modifié. Le sol sur lequel se sont installés les premiers habitants du site est composé d'une argile orange et compacte contenant des concrétions ferrugineuses. Il est généralement recouvert, sur une faible épaisseur, de sols gris et mal drainés, plus pauvres que ceux de la plaine d'inondation. C'est cette argile rouge, la lal mati, utilisée pour sa couleur, que les constructeurs de Garh vont souvent chercher hors du site pour ne pas être contraints de traverser toute l'épaisseur des couches archéologiques afin de la trouver.

5 La plus ancienne occupation attestée du site remonte à la fin du $\mathrm{IV}^{\mathrm{e}}$ siècle ou au début du III ${ }^{e}$ siècle avant J.-C. (datation 14C) (Bernard, Boussac, Breuil \& Salles 2001). Les fouilles ont montré que, si sa densité d'occupation a été plus ou moins distendue suivant les périodes, le site a sans doute été habité de façon relativement continue jusqu'au XIII ${ }^{e}$ siècle de notre ère au moins. Les techniques de construction ont beaucoup évolué au cours des temps - maisons de terre crue, voire de briques crues et rempart de briques cuites de gros modules durant les périodes les plus anciennes, puis édifices de briques cuites (peut être parfois mixte: fondation et soubassement de briques avec élévation et sols en terre). Les briques sont de modules très différents suivant les époques et leur taille tend à se réduire. La très grande majorité des constructions découvertes (le plus souvent en fondations) -murs du rempart exclusétaient bâties en briques de réemploi généralement fractionnaires et en terre. Quelques bâtiments monumentaux sont faits de briques complètes et comportent des éléments en pierre (montants, linteaux et colonnes, principalement). La fouille montre, à toutes les périodes, les très nombreuses fosses d'extraction ou de pillage qui ont servi à la construction.

6 On comprend bien, aussi, en stratigraphie, comment on détruit, récupère, surélève, pour bâtir de nouveau. C'est ce mélange, que d'autres ont déjà préparé, et qui, de plus, est mixé de tessons et de nodules de briques, qui sert aujourd'hui à la construction des maisons de Garh.

7 Exemple frappant de réemploi des matériaux : les murs bâtis en briques archéologiques (très souvent des murs d'enclos) illustrent fréquemment l'évolution des modules de briques. Le mur est bâti au fur et à mesure de l'extraction des briques, remontant le temps et affichant, à l'inverse, la stratigraphie du site: les plus petits modules se retrouvent en bas du mur, tandis que les briques les plus grosses sont au sommet.

8 Le rempart -en fait, une série de remparts successifs recouverts de terre, formant une surélévation en forme de digue-, qui encercle totalement le site, subit aujourd'hui encore, malgré le classement du site, une dégradation intensive due au pillage de ses briques, grandes et complètes.

9 D'autres types d'objets archéologiques servent également à la construction : ici, un linteau de pierre sert de marche vers le dighi (réservoir d'eau); là, une pierre en guise de seuil. 


\section{Garh, un habitat concentré}

10 Mahasthangarh est constitué de deux parties : l'une basse, Mahasthan, comportant un village avec des maisons en bambou et un bazar; l'autre haute, Garh, le site archéologique, dont l'habitat est concentré et environné de terres agricoles ou laissées en friches fouillées chaque année par les archéologues. En outre, s'y trouvent un sanctuaire et une grande mosquée. C'est sur Garh qu'a porté cette étude, car c'est dans ce seul village que sont construites les maisons en terre (fig. 1 et 2).

11 Mahasthangarh est bien considéré comme un village (gram) malgré une population totale de 3281 habitants et 496 feux en 1991. Sa surface totale est de 751 acres, soit 300,40 ha (Bangladesh Population Census 1991 : 122). Les deux parties, hautes et basses, sont à peu près équivalentes puisque les archéologues ont estimé à 165 ha l'espace de Garh.

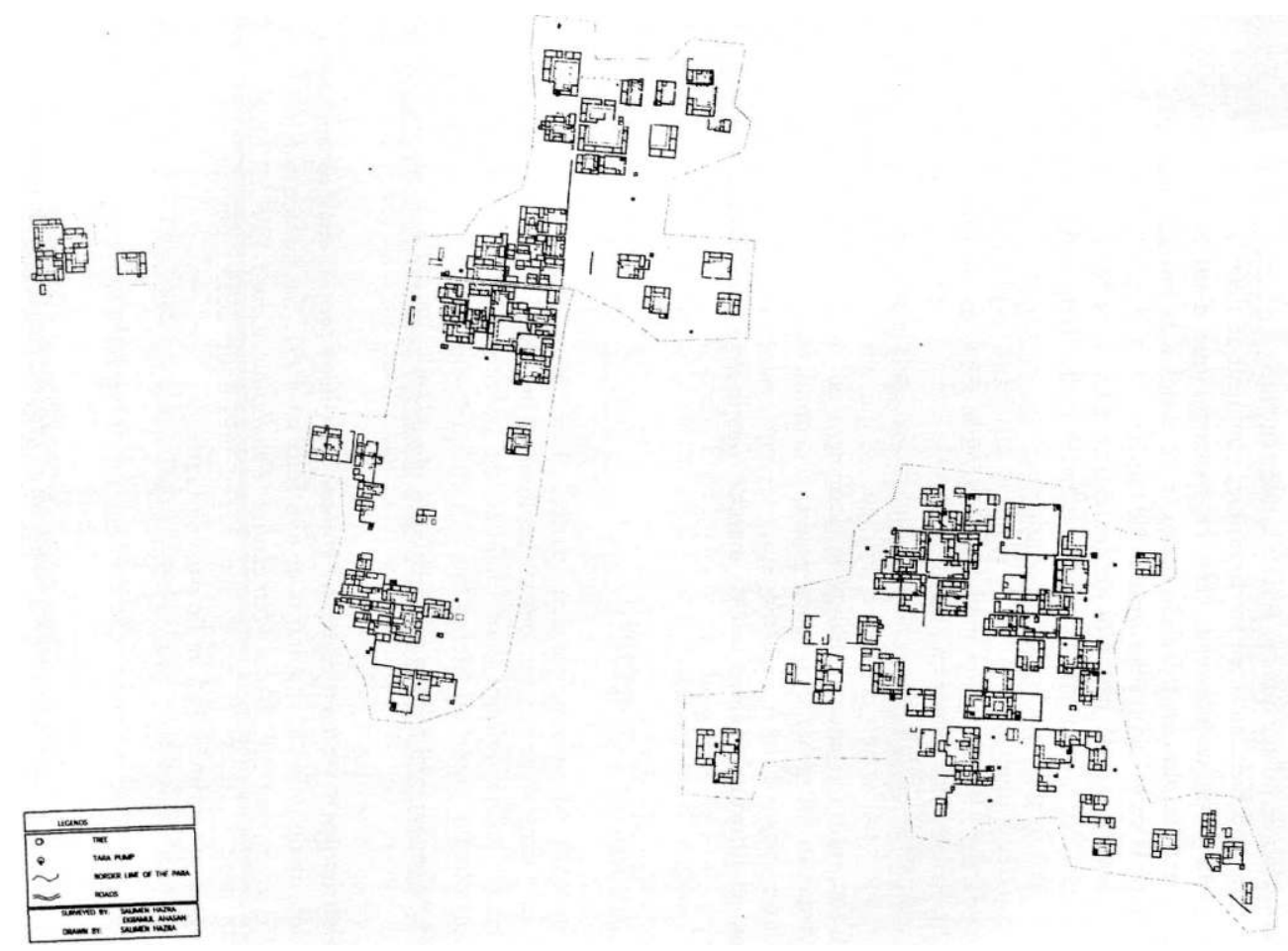

Figure 1. Les trois quartiers (para) étudiés à Garh 


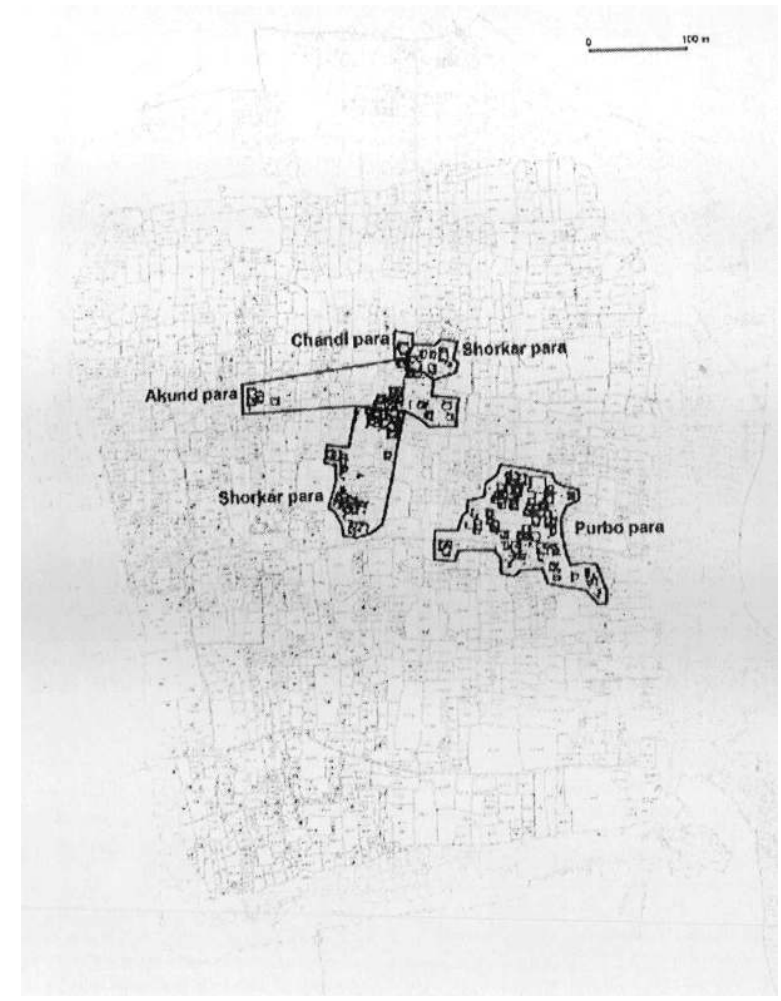

Figure 2. Limites de para de Mahasthangarh (surveyed by Saumen Hazra and Ekrawlil Ahasan) fleuves qu'il prend une forme dispersée ${ }^{7}$. À Garh, les unités d'habitation se côtoient, dans la plupart des cas, par un mur mitoyen, un des côtés étant laissé libre pour le passage des eaux usées. Le regroupement des maisons s'explique certes par un manque de terrain, mais aussi par la crainte des mauvais esprits (bhut), susceptibles d'occuper une habitation isolée et de "posséder" ses habitants, tout particulièrement les femmes. disposant d'un large terrain n'occupent pas forcément tout cet espace pour y habiter. Une partie peut être occupée par des toilettes (d'environ $1 \times 1 \mathrm{~m}$ ) qu'ils préfèrent mettre à distance de l'unité d'habitation; les murs sont construits en ciment ou en feuilles de bambou, et l'on évite de les diriger vers le sud-ouest, la direction de la Mecque. Seules les familles qui ont des moyens financiers conséquents en possèdent. Les autres vont dans «la jungle», pourtant peuplée de mauvais esprits (bhut). Car, malgré un voisinage de proches parents, les toilettes sont rarement partagées. Une pompe à eau potable commune, offerte il y a quelques années par le gouvernement, peut également être implantée sur un terrain en propriété privée. Le reste de l'espace attenant à l'unité d'habitation peut être consacré à une étable, une aire de battage, une 
meule de foin, voire une bambouseraie ou un couvert d'arbres qui sont exploités pour la vente. Il arrive même qu'un espace plus large serve à enterrer les morts de la famille élargie (gusti) ${ }^{8}$, ou lorsqu'il est laissé vide, à se réunir. À Garh, il n'existe en effet aucune place publique. Quelques chefs de famille possèdent également une mare en propriété privée', bien que les voisins puissent en avoir l'usage pour laver leur linge ou leurs animaux et pour y prélever la terre de construction. Les propriétaires, et eux seuls, l'emploient aussi pour l'élevage des poissons ou la pêche. En général, elle est située à proximité de l'unité d'habitation et peut même prendre l'aspect d'une simple cavité, non entretenue en dehors de la saison des pluies. Un proverbe de Khona recommande d'ailleurs de construire la maison près de la mare ${ }^{10}$.

Les seules bâtisses qui ne soient pas des unités d'habitations sont les petites épiceries, érigées en bordure des chemins, et les mosquées, voire les écoles coraniques ou les écoles d'organisations non gouvernementales (ONG), qui ne sont pas toutes en terre.

\section{La maison à cour}

16 L'unité d'habitation est nommée bari. Elle comprend une ou plusieurs cellules d'habitation (ghor), - pourvues chacune d'une véranda- réparties autour d'une cour (uthan ou anguena) et fermées par un mur d'enceinte, presque toutes d'un seul niveau. Ce modèle de maison à cour est très répandu dans l'ensemble du continent indien (Noble 1987 ; Mitra 1966). Pour la grande majorité des habitants de Garh, le terrain est la propriété du chef de famille ; rares sont les unités d'habitation en location. D'après la structure résidentielle étudiée dans trois quartiers qui comprennent 139 unités d'habitation, deux seulement correspondent à une famille étendue en indivision. Les autres sont soit des familles nucléaires, soit des familles étendues mais dont les fils sont autonomes (bhinnu) ${ }^{11}$.

17 L'espace habité est surélevé par rapport aux terres agricoles, même celles sur lesquelles on cultive les légumes, les plus hautes, dites bhita, en comparaison des terres basses (nitcu ou nama) dévolues à la culture du riz.

18 La cour constitue la partie centrale de l'unité d'habitation, où ont lieu, à la saison sèche, la plupart des activités quotidiennes ainsi que certains travaux agricoles comme l'étuvage et le vannage du riz, le tri et la pesée des légumes, etc. C'est souvent un espace ombragé, car les habitants ne manquent jamais de planter un ou deux arbres sitôt la construction terminée. Autour, sur un, deux ou trois côtés, se trouvent les cellules d'habitation et l'étable qui sont soit accolées, soit espacées (photo 1). 


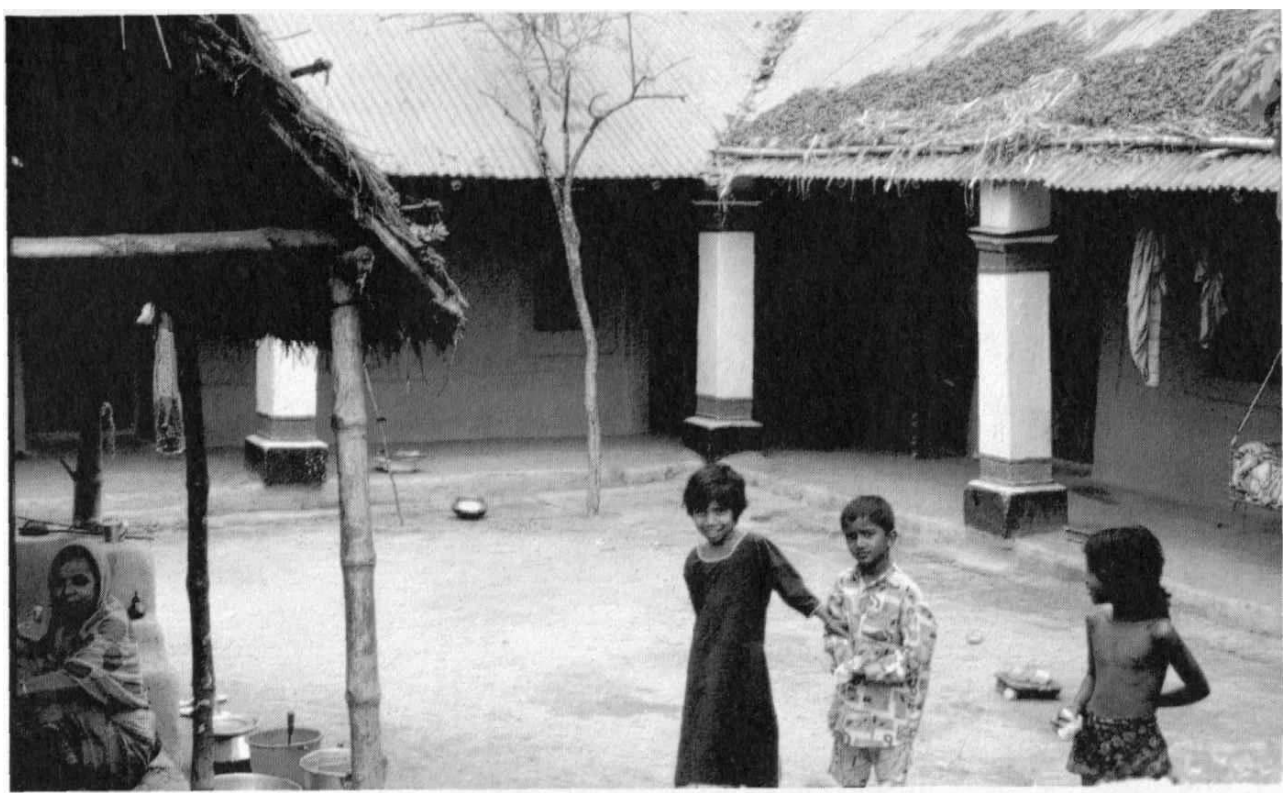

Photo 1. Intérieur de l'unité d'habitation (bari)

19 Chaque cellule d'habitation est de forme parallélépipédique et, dans la majorité des cas, comprend une seule pièce, de sorte que les cellules ne communiquent pas, afin de préserver l'intimité. Ainsi, pour aller d'une cellule à une autre, faut-il passer par la cour ou la véranda. Lorsqu'une cellule comporte deux pièces, c'est qu'y habite une famille peu fortunée et dont cette seule cellule, pourvue d'un mur de partition intérieur, abrite un proche parent adulte du chef de famille. La cellule est alors dotée d'une seule ouverture extérieure pour les deux pièces.

20 Le nombre de cellules autour de la cour est variable; la moyenne étant de trois par unité d'habitation. Cela dépend de la surface du terrain disponible, de la taille de la famille (notamment du nombre de couples) et des moyens financiers; une famille nucléaire possédant trois cellules, par exemple, peut ainsi répartir ses occupants et en destiner une aux ustensiles de cuisine et aux réserves. La position de ces cellules ne répond ni à un impératif absolu d'orientation, ni à aucune croyance. Mais les habitants favorisent l'orientation au sud, qui permet une bonne ventilation intérieure ${ }^{12}$. Aucune orientation n'est privilégiée non plus quant à l'entrée principale de l'unité d'habitation et à l'étable. Généralement de petite taille, celle-ci peut contenir une ou deux vaches ${ }^{13}$ et se présente de l'extérieur comme n'importe quelle cellule tant on prend soin de l'apparence des constructions. Ce soin, constaté à Garh, contredit les données de D. M. Hasan (1985 : 46) qui l'a remarqué dans les seules maisons des hindous; pour lui, cela résulterait du respect attribué aux vaches et au feu, ce qui donnerait une maison plus nette et plus rangée que celle des musulmans.

21 L'étable ne comporte cependant aucune fenêtre, contrairement aux cellules d'habitation qui en ont sur le devant, côté cour, et à l'arrière pour permettre une bonne ventilation des constructions. Les fenêtres sont parfois pourvues de volets en bois ou simplement obturées par un tressage de feuilles de bambou ou même par un textile. Les habitants peuvent donc communiquer d'une maison à l'autre, ou entendre les conversations des voisins. Ils ne s'en plaignent jamais et le vis-à-vis entre hommes et femmes est toutefois évité afin de respecter les règles de l'honneur qui conditionnent le comportement des uns et des autres. En fait, la partition entre hommes et femmes n'est 
pas stricte ${ }^{14}$. Faute de moyens financiers, rares sont les familles qui possèdent une cellules d'habitation réservée aux hôtes masculins (baîtak ghor). Dans ce cas, la porte est tournée vers l'extérieur de l'unité d'habitation afin que les hommes ne pénètrent pas dans l'espace familial. Faute de moyens encore, la majorité des habitants reçoivent leurs hôtes à l'intérieur. Les proches parents et les femmes peuvent être conviés au sein d'une cellule d'habitation; les autres visiteurs sont invités à s'asseoir sous la véranda, hommes et femmes se côtoyant. Les femmes alors se couvrent la tête de leur purdah, l'extrémité du voile de leur sari.

La véranda, tournée vers la cour, prolonge la cellule d'habitation. Sa base est constituée d'une terrasse qui se trouve à peu près au même niveau que l'entrée des cellules. Elle a de multiples fonctions, tout spécialement en période de pluies et de fortes chaleurs: elle protège les murs de la pluie, c'est un lieu d'activités et même un endroit pour dormir lorsqu'il n'y a pas assez de place pour toute la famille. Le poulailler y trouve sa place, ainsi que les rares pilons à décortiquer le paddy (dheki).

L'espace dévolu à la cuisson des aliments (hatsel) est situé dans la cour, au centre ou près du mur d'enceinte, peu importe. Mais la bouche du foyer (mukh) par laquelle celuici est ravitaillé en combustible, doit toujours être dirigée vers l'est. Une autre orientation apporterait du malheur dans la maison. L'espace peut être ou non couvert d'un toit. Chaque unité domestique autonome (bhinnu) possède son propre foyer (tcula). Car celui-ci représente l'unité économique et l'unité de consommation. Autrement dit, lorsqu'un fils marié devient autonome et qu'il conserve ses propres revenus, son épouse construit un foyer. Dès lors, celle-ci cuisine pour sa propre famille et le couple mange seul ${ }^{15}$. Ces foyers situés dans la cour et rassemblés, de telle sorte que les femmes cuisinent côte à côte, ne servent qu'à la saison sèche. Le reste de l'année, chaque épouse construit un foyer mobile et cuisine dans sa cellule d'habitation ou sur la terrasse de sa véranda.

Lorsque l'unité domestique a les moyens de se procurer une pompe à eau potable, elle la fait installer dans un coin de la cour (peu de familles possèdent un puits). De préférence, celle-ci sera enclose par un mur en ciment afin de protéger ses habitants lorsqu'ils font leurs ablutions. Le mur d'enceinte de l'unité d'habitation doit permettre de respecter l'intimité des familles. Or, il ne remplit pas toujours cette fonction car cela dépend de sa hauteur. Au mieux, il mesurera $2 \mathrm{~m}$, au pire, quand il n'est pas absent, $1,60 \mathrm{~m}$; il sera en partie éboulé et son entrée ne sera pas pourvue de porte. Dans l'idéal, il doit être élevé, fait d'un mur aveugle et fermé lorsque ses habitants dorment ou sont absents. Il convient de noter qu'aucune entrée en chicane n'existe à Garh, aussi chacun peut-il porter son regard à l'intérieur de l'unité d'habitation. Il est rare de trouver deux entrées dans cet espace familial. Hommes et femmes pénètrent donc par la même porte. Lorsqu il y en a deux, l'une d'elles sert à communiquer d'une maison à une autre, les voisins étant parents directs.

\section{Les techniques de construction}

Les maisons en terre crue (dewaler matir ghor) constituent la majorité des habitations de Garh. Les autres sont faites de briques cuites industrielles qui sont une marque d'enrichissement de la famille. L'une d'elle est en tôle galvanisée. D'après ses propriétaires, ce choix résulte de l'interdiction de creuser le sol pour obtenir de la terre. 

que soit l'endroit où ils creusent ${ }^{17}$, ils recueillent de la terre mais aussi des morceaux de briques cuites anciennes. Ce sont ces briques que les habitants collectent régulièrement même s'ils n'ont pas de projet immédiat. Elles servent parfois à renforcer les vérandas ou à fabriquer des piliers. Elles sont alors noyées dans la terre. Ponctuellement, elles servent aussi de renfort aux drains et à ce qui touche à la circulation de l'eau ou encore à protéger de la pluie : une brique peut servir de marche pour accéder à la véranda par exemple ou bien, on peut la poser sous les pieds des lits en bois pour les préserver de l'humidité.

Mais la brique sert avant tout pour les fondations ainsi que pour le mur d'enceinte de l'unité d'habitation. C'est d'ailleurs la raison de la faible hauteur de certains murs : le chef de famille n'avait pas assez de briques archéologiques à sa disposition, lorsqu'il en trouvera d'autres, il continuera d'élever son mur. Comme l'usage de ces briques est interdit, les habitants les utilisent là où elles sont cachées (dans les fondations, la terrasse) ou en les enfouissant dans la terre (pilier des terrasses par exemple), et là où il 
serait le moins grave d'être obligé de démolir. C'est pourquoi ils ne les emploient jamais pour les murs de maison, mais seulement pour le mur d'enceinte.

Les fondations sont une partie importante de la cellule d'habitation. Certes pour la solidité du bâtiment, mais aussi pour sa représentation symbolique. Elles incluent le volume de terre qui s'élève à peu près au niveau de la terrasse, c'est-à-dire à environ $30 \mathrm{~cm}$ de hauteur. De l'extérieur, cette partie forme une semelle par rapport aux murs de la maison. Elle est nommée bhita. Ce terme, qui signifie "fondation ", évoque également la terre des ancêtres : sa largeur, sa solidité, évoquent la valeur et la longue lignée d'ancêtres ayant vécu dans la maison.

Les fondations sont faites d'un mélange de terre sèche et de morceaux de briques archéologiques tassées aux pieds. Qu'il y ait une ou plusieurs cellules d'habitation à construire, on fait ce travail en une seule fois. Ensuite, une certaine quantité de terre est entassée près du chantier où elle est humidifiée, puis foulée aux pieds, le plus souvent par les femmes et les enfants, et enfin laissée au repos pendant trois jours. La terre étant alors compacte, elle est foulée à nouveau par les hommes. L'un d'eux la découpe ensuite à l'aide de sa houe, un second en fait des boules (matir got) d'environ $20 \mathrm{~cm}$ de diamètre et les lance à un troisième qui commence à monter le mur. Ce dernier entreprend la construction à partir du côté droit de l'emplacement de la porte et continue sur les quatre côtés.

Chaque niveau de construction (bat) fait à peu près $30 \mathrm{~cm}$ de hauteur. Celui qui élève le mur est en position accroupie sur ce mur. Après avoir reçu la boule de terre, il la projette d'une hauteur de 30 à $40 \mathrm{~cm}$ afin de la tasser. C'est la technique de la bauge (photo 2).

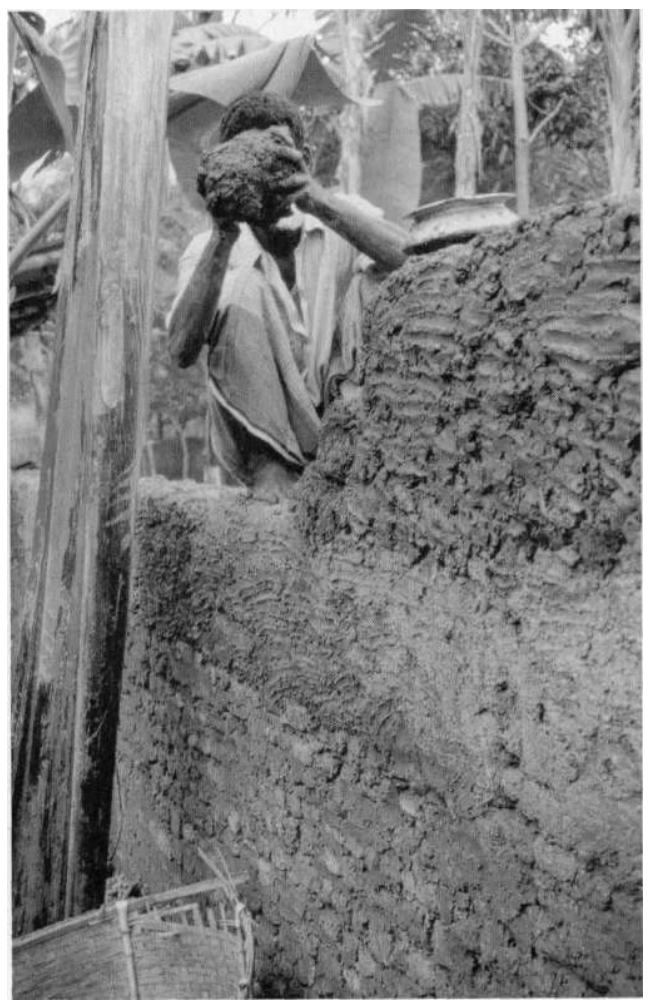

Photo 2. Élévation d'un niveau de construction par la technique de la bauge

Il place et tasse plusieurs de ces boulettes à la main et aligne les bords et la surface du mur, toujours à la main. Aucun bourrelet débordant qui permettrait d'emboîter les 
niveaux n'est réalisé. Lorsqu'un niveau est terminé, il faut attendre une semaine de séchage avant d'en commencer un deuxième. L'homme prévoit l'emplacement de la porte et des fenêtres en laissant des espaces vides et non en construisant sur toutes les faces et en creusant par la suite. Il arrive cependant que l'on retaille les ouvertures au burin lorsqu'une cellule d'habitation est rajoutée, accolée à une autre, afin d'aménager une circulation intérieure entre les deux espaces. De même, le constructeur anticipe l'espace des niches intérieures (il y en a deux, en général) en réduisant l'épaisseur du mur. Portes et fenêtres sont surmontées d'un linteau (muka) fait de plusieurs bambous ${ }^{18}$ liés par de la ficelle, qui sont placés au moment de la construction. Le contour des ouvertures est le plus souvent légèrement saillant sur une trentaine de centimètres de large et 1 ou $2 \mathrm{~cm}$ d'épaisseur. Cet aménagement architectonique (il permet de renforcer la structure à ses endroits les plus vulnérables : les arêtes) est habituellement programmé, dès la pose des premières assises de terre, par un décrochement. Son rôle est également esthétique : il est presque systématiquement coloré autrement que les murs (photo 3).

Comme nous l'avons dit, la surface des fondations est plus large que celle des murs, lesquels sont légèrement plus épais à la base qu'à l'extrémité : de 60 à $40 \mathrm{~cm}$ environ. L'arête des murs n'est pas renforcée.

La surface des cellules d'habitation est en moyenne de $27 \mathrm{~m}^{2}$ avec une hauteur de $3,60 \mathrm{~m}$ au plafond. Rares sont les cellules d'habitation à deux niveaux ${ }^{19}$. Lorsqu'il y en a un, il est peu élevé et sert à emmagasiner les produits agricoles. Les réserves sont habituellement entreposées entre la charpente et le plafond dans lequel une petite ouverture est alors pratiquée, ou dans une partie d'une cellule d'habitation, ou encore sous les lits. Ces maisons à deux niveaux appartiennent à ceux qui ont de vastes terres agricoles $^{20}$.

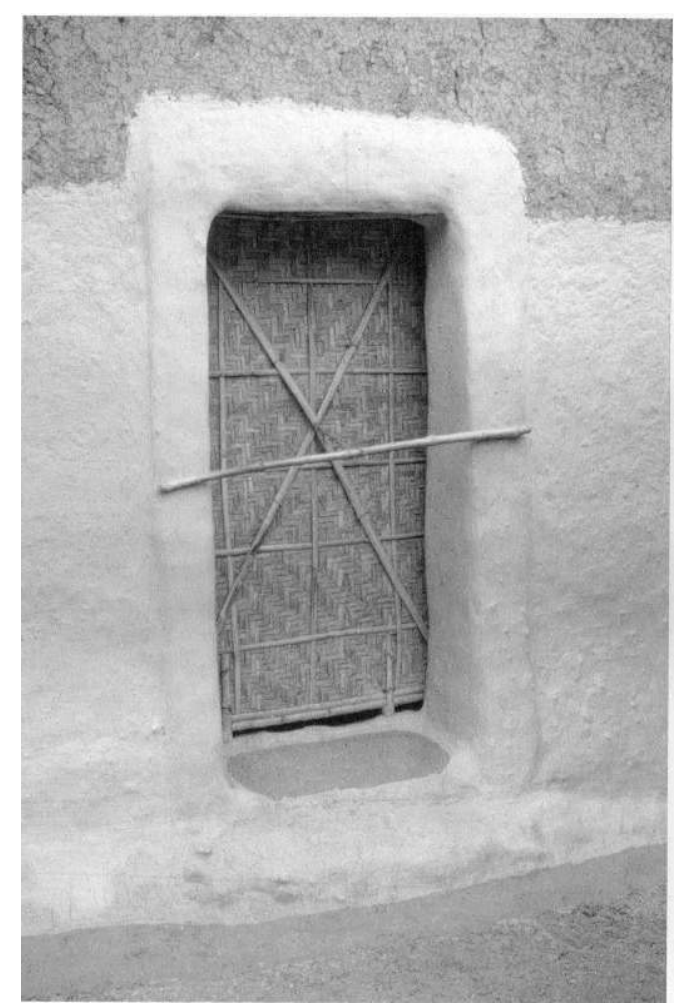

Photo 3. La partie basse du mur de maison est recouverte d'un enduit. La porte est en bambou 
Arrivés au plafond (tala), les constructeurs placent 5 chevrons de bambou (tir) dans le sens de la largeur selon un espacement d'environ $60 \mathrm{~cm}$; deux autres (kaklatir) sont alignés dans le sens de la longueur, auxquels s'en ajoute un (kanetir) à chaque coin. Le dessus du plafond est recouvert d'un lattis (bata) de bambous sur lequel est étendue une couche de terre mi-argileuse, mi-sableuse appelée doac mati ${ }^{21}$, que l'on récolte près de la rivière. C'est la terre accumulée qui retient les bambous. Ces derniers ne sont pas toujours traités pour éviter les insectes. Parfois, ils sont laissés à tremper pendant deux semaines dans une mare. Dans le meilleur des cas, les chevrons sont recouverts de goudron. Jusqu'à ce niveau, la construction peut être réalisée par des non-spécialistes -bien que chaque habitant de Garh ait une certaine expérience de ce travail (photo 4).

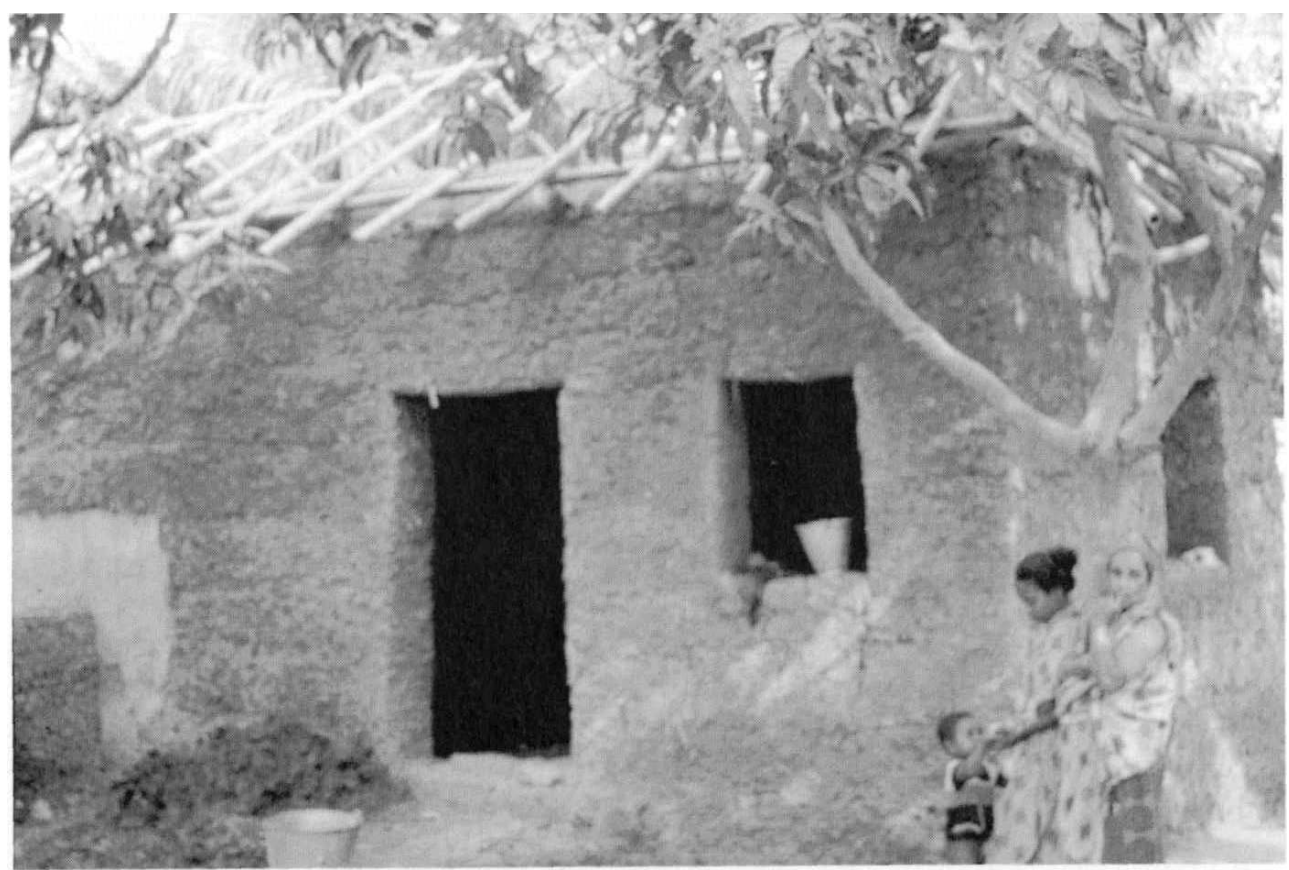

Photo 4. Cellule d'habitation en construction : les artisans réalisent la charpente

Le parallélépipède achevé, la famille entreprend la réalisation de la terrasse (poeta) qui borde la cellule d'habitation. C'est un travail équivalent à celui des fondations : des couches de terre sont étalées en alternance avec des couches de briques archéologiques, puis damées aux pieds. Lorsque la famille est un peu aisée, c'est la première structure qu'elle fera réaliser en ciment car elle ne protège que partiellement la cellule d'habitation. La terrasse, à 30 ou $40 \mathrm{~cm}$, est légèrement surélevée par rapport à la cellule. De sorte qu'il y a un petit emmarchement pour passer de l'une à l'autre. Le seuil (dwar, ce qui signifie également " porte ») sera renforcé par une barre de bois qui déborde de la terrasse et empêche ainsi l'eau de pénétrer dans la cellule d'habitation en cas de fortes pluies. La largeur de la terrasse est d'environ $1 \mathrm{~m}$. L'étape suivante consiste à élever deux ou trois piliers qui permettront de maintenir la toiture de la terrasse. Ces piliers peuvent être réalisés uniquement en terre ou en briques intégrées dans la terre de manière à être totalement dissimulées.

La toiture (tcal) est à double pente (do cala) mais les propriétaires ne couvrent pas toujours les côtés du pignon par souci d'économie. Toutes les toitures sont maintenant en tôle galvanisée ondulée car ce matériau peut durer jusqu'à 20 ans. Jadis, les bâtisses étaient couvertes de chaume qui ne résistait que deux années. Le chaume subsiste encore sur quelques abris de foyers. Apparemment, il n'y a jamais eu de couvertures en 
tuiles d'argile cuite, car selon certains habitants, ce matériau est trop lourd. Pourtant, on peut en voir posées sur quelques murs d'enceinte. Le problème du toit en tôle est qu'il accumule la chaleur. Aux dires des résidents, ce problème serait atténué par le plafond en terre: l'espace entre celui-ci et la toiture permettant une certaine ventilation.

41 Trois ou quatre artisans spécialisés dans la confection des toitures sont engagés par le chef de famille. En général c'est ce dernier qui apporte les matériaux (bois, bambou, tôle). Les artisans arrivent avec leurs outils (marteau, scie, clous, ficelle ou fil de fer, goudron éventuellement). Ils commencent le travail lorsque le plafond est assez sec, deux semaines après qu'il a été terminé, par la réalisation de la ferme. Celle-ci est faite de chevrons en bois qui forment l'entrait et sont fixés sur le plafond, puis de bambous (parfois recouverts de goudron juste avant leur mise en place) formant l'arbalétrier. Ces derniers sont ficelés entre eux et au bois, ou quelquefois cloués ; mais on ne devrait pas clouer le bambou : cela risque de le fendre (Lopez 1982-83:100) ${ }^{22}$. Sur l'arrière de la bâtisse, la tôle forme un débord de toiture, laquelle est soutenue par un chevron en bambou à chaque extrémité. À l'avant, elle est fixée sur deux ou trois longs bambous (les arbalétriers) et forme une avancée qui constitue le toit de la véranda. Le débord et la véranda assurent ainsi une bonne protection des murs au moment des fortes pluies. Les bambous qui retiennent l'avancée de la véranda sont appuyés sur des piliers ancrés sur la terrasse. Les artisans effectuent ce travail en une journée.

42 La construction s'achève par la pose d'une plinthe (pirali) qui court sur trois côtés (à l'exclusion de celui de la terrasse). Cette plinthe est réalisée par le chef de famille. Elle a une vingtaine de centimètres d'épaisseur et s'élève à une hauteur égale. Sa fonction est de renforcer la base de la maison. Elle est remise en état chaque année.

Pour réaliser une cellule d'habitation il faut compter une durée de trois à quatre mois. Il va sans dire qu'une construction ne peut être interrompue d'une année à l'autre car les murs fondraient sous les pluies de la mousson. La bâtisse achevée, il faut laisser sécher les murs en l'état pendant un an. À cet effet, la ventilation opérée par les fenêtres pratiquées dans les deux murs de longueur sont très utiles. C'est à l'issue de cette année que l'épouse passera un enduit à l'intérieur et à l'extérieur des murs et exécutera les décors autour des fenêtres, de la porte et des niches, ainsi que sur les piliers de la terrasse.

44 La dernière étape de la construction concerne le mur d'enceinte, quand il s'agit d'une unité d'habitation. Comme nous l'avons dit, celui-ci est, dans la plupart des cas, fait de morceaux de briques archéologiques montés sur un mortier de terre. Son épaisseur est de $30 \mathrm{~cm}$ environ.

Une fois terminée, l'habitation ne peut être occupée par ses habitants sans que soit célébré un rituel social et religieux. Il s'agit d'une prière (milad) conduite par un religieux (munsi), suivie d'un repas où sont conviés parents et voisins. À cette occasion, aucun animal n'est sacrifié ; ce n'est pas la coutume de verser le sang pour inaugurer une nouvelle maison.

\section{Le coût de la construction}

Les dépenses consenties par un chef de famille pour bâtir une cellule d'habitation varient selon qu'il emploiera un artisan et son équipe, ou aura recours à des journaliers 
et effectuera une bonne part des tâches avec son épouse et ses enfants. Les travaux de fondations, ceux qui concernent les murs et le plafond sont rémunérés $150 \mathrm{tk}^{23} \mathrm{par}$ coudée (hat), pour un artisan, lequel décide lui-même du nombre d'ouvriers à engager. Pour une cellule d'habitation de $6 \mathrm{~m}$ (soit 15 hat) sur 4,5 m (11 hat), le prix sera calculé en fonction d'une longueur et d'une largeur; c'est-à-dire : $15+11=26$ hat x $150 \mathrm{tk}=3900 \mathrm{tk}$. La hauteur de la bâtisse n'est pas prise en compte car elle est standard : 9 hat $(3,60 \mathrm{~m})$. Trois repas sont offerts aux ouvriers avec du riz, de la viande et des sucreries :

-à l'issue de la construction des fondations (bhit dawa: bhit, lit. fondations; dawa, construction);

- à l'achèvement du linteau de la porte (muka para : muka, lit. linteau ; para, placer) ;

- lorsque le plafond est terminé (tcal para : lit. placer le toit).

Pour la réalisation de la toiture, les ouvriers sont payés $80 \mathrm{tk}$ par personne et par jour, auxquels s'ajoutent deux repas. En outre, le chef de famille doit acheter le bois, les bambous, éventuellement payer la terre de construction et son transport. Cela reviendrait à environ 7500 tk. La porte en bois coûte $1500 \mathrm{tk}$, les fenêtres avec volets en bois 600 tk chacune, 1000 lorsqu'elles ont des barreaux en fer forgé. Elles sont réalisées par des artisans menuisiers, en bois de jaquier (kathal), qui traitent avec des forgerons (hindous) pour la fabrication des fenêtres en fer forgé. Au total un chef de famille dit devoir dépenser $100000 \mathrm{tk}$ pour ce type de construction. Cette somme nous paraît élevée par rapport à notre brève estimation totale: environ 20000 tk sans compter le coût du mur d'enceinte qui est souvent insignifiant. Même si on ajoute le prix du terrain (le propriétaire ne possède aucune facilité sanitaire) d'une surface de 2 cotok, soit $1886 \mathrm{~m}^{2}$, à raison de $4000 \mathrm{tk}$ par cotok en 2001, soit 8000 tk, nous sommes loin de la somme indiquée.

\section{Entretien et décor de la maison}

L'entretien de la maison est la condition essentielle de sa durabilité, et son décor y participe même s'il ajoute une dimension esthétique. Un an après la construction, l'épouse du chef de famille entreprend les finitions de son habitation. C'est en effet un travail exclusivement féminin, comme tout travail d'entretien et de décoration; et les femmes le font seules, la plupart du temps. Mais c'est l'époux qui se procure les terres destinées aux revêtements de mur et de sol. Pour les murs, il doit obtenir une terre limoneuse (poli mati) qu'il achète au bazar de Mahasthan. En ce qui concerne les sols, un enduit jaune/orange (à base de latérite ?), appelé lal mati, est récolté à une certaine profondeur du sol, à Garh même ou dans d'autres villages peu éloignés, à Gokul ou à Matura.

Les décors sont réalisés à partir d'une terre de couleur rouge (ghri mati) acquise au bazar de Mahasthan. Chaque épouse dispose toujours de ses réserves de terre dans un coin de la maison.

La première activité à l'issue de l'année de construction est le revêtement de poli mati sur l'ensemble des murs intérieurs et extérieurs, ainsi que sur les sols jusqu'à la terrasse. La terre est diluée dans l'eau, cette solution étant nommée losna. À l'exception de la terrasse, dont le sol est ensuite recouvert par l'épouse une fois par semaine, avec un mélange de lal mati, de bouse de vache et d'eau, les autres éléments architecturaux 
ne reçoivent cette préparation de poli mati qu'une à deux fois par an (photos 5 et 6). Les failles éventuelles qui se présentent dans le mur sont recouvertes de poli mati un peu plus épaisse, tout comme les murs extérieurs d'ailleurs. La plupart du temps, ces derniers ne sont pas protégés par l'enduit sur toute la surface, mais seulement jusqu'à une hauteur de 1,50 m environ. Cette action de revêtir de terre une surface se nomme lapa.

51 Nous avons déjà évoqué la question de la plinthe (pirali). Son état est vérifié chaque année par le chef de famille qui, si elle est endommagée, la renouvellera. Elle est fabriquée exclusivement en terre, mouillée et foulée aux pieds, puis placée tout le long de la bâtisse à l'aide de la houe et enfin, tassée et lissée à la main.

Dans la plupart des cas les décors sont appliqués sur les murs qui sont sous le plafond, autour des niches (almari) toujours situées à l'intérieur de l'habitation, autour des fenêtres et des portes faisant face à la cour, et sur les piliers. On les exécute avec cette terre ghri mati qui est mélangée à de l'argile et de l'eau. Cette solution, qui disparaît sous l'effet de la pluie, est peu employée sur les piliers de la terrasse. Ceux-ci sont plutôt revêtus de peinture industrielle.

Les plus riches décors sont ceux qui se situent sous le plafond. Là, l'épouse s'exprime selon sa fantaisie en imprimant des volutes ou des motifs floraux. Elle peut reproduire ces décors autour des niches ou se limiter à dessiner une large bande sur leurs contours. Bande qui se retrouve tout autour des fenêtres et des portes, y compris la porte de l'étable. La porte principale de l'unité d'habitation est rarement décorée, sauf si elle est en fer et disposée sur un mur en ciment. Elle dénote alors la richesse de ses propriétaires. Ces décors montrent combien les habitants de Garh prennent soin de leurs maisons. Celles-ci présentent toujours un aspect très plaisant, de bon entretien, lorsque l'on pénètre à l'intérieur. Peu d'habitations sont privées de décor ou mal entretenues. Quand c'est le cas, elles appartiennent à des familles particulièrement pauvres, surtout à des personnes âgées n'abritant plus d'enfant ou bien des femmes veuves ou divorcées qui sont dans l'obligation de travailler en dehors du village. 


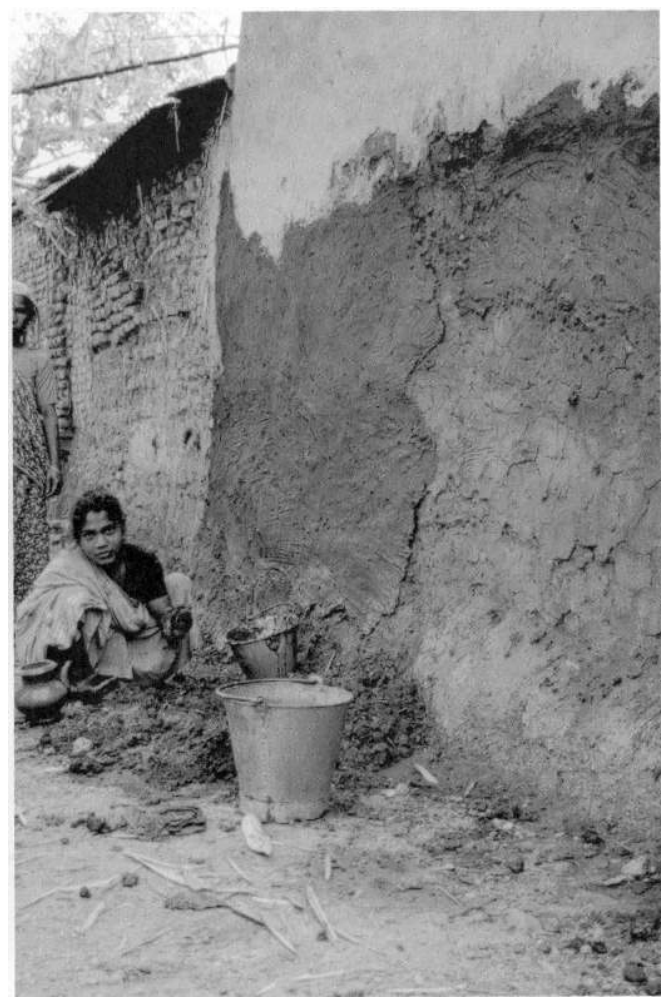

Photo 5. L'épouse recouvre d'un enduit le mur extérieur de sa maison

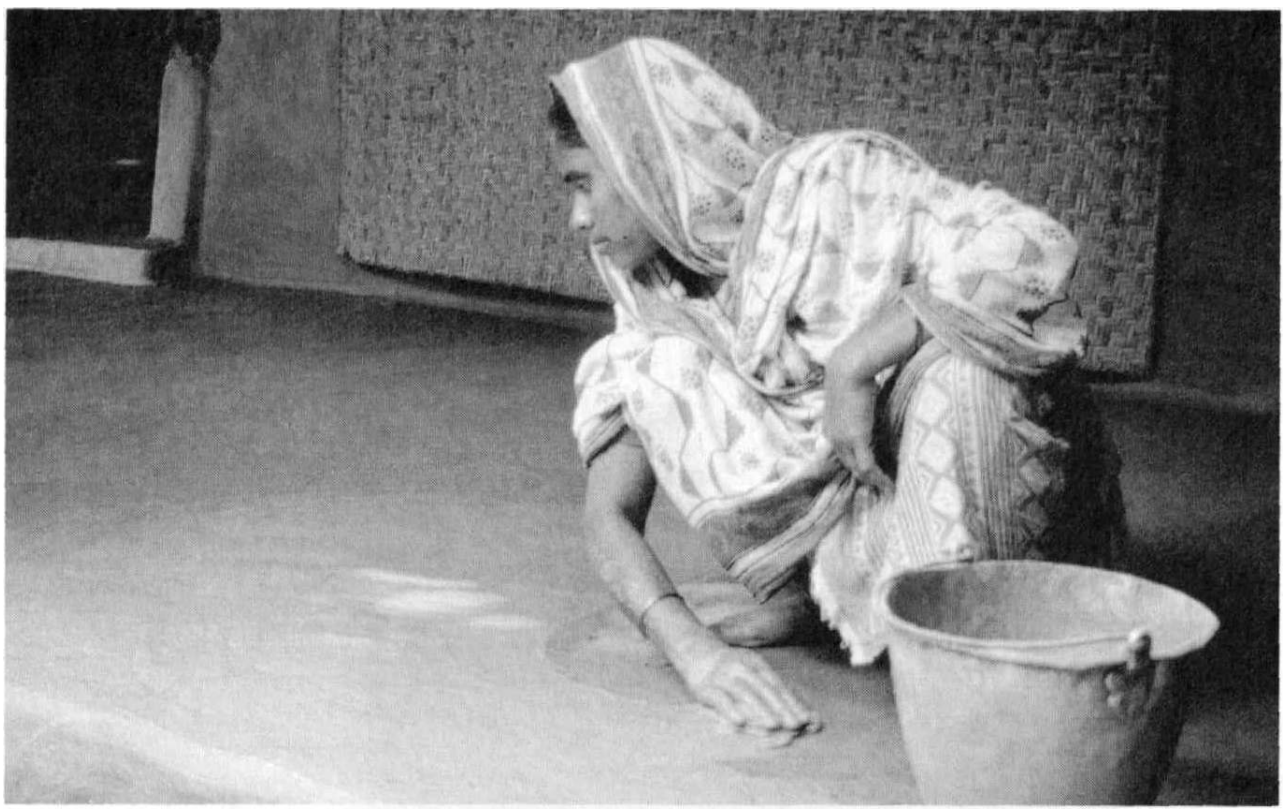

Photo 6. L'épouse applique un enduit de terre et de bouse de vache sur le sol de la terrasse

54 Un autre type de décor est réalisé dans des conditions exceptionnelles : par exemple pour un mariage. À cette occasion, les femmes et les fillettes de la maison appliquent une couche de poli mati sur les murs attenant aux terrasses. Puis, avec une branche ou un objet pointu, elles gravent des motifs variés (fleurs, volutes...). Ainsi, lorsqu'on pénètre à l'intérieur d'une unité d'habitation ainsi décorée, on sait qu'un mariage est en préparation ou vient d'avoir lieu. 


\section{L'habitat rural} et humide. En effet, les murs des cellules d'habitation sont très épais, larges d'une coudée, ce qui empêche une bonne ventilation intérieure et créé une isolation thermique ; cela malgré le nombre d'ouvertures. En revanche, en cas de forte tempête ou de cyclone, ces maisons conservent une bonne assise. Les habitants de Ghar pourraient aussi utiliser le bambou dans leur construction, car celui-ci pousse partout. Mais ce type de maison, répandu au Bangladesh, est peu résistant et d'une durée de vie réduite. Ce choix de la terre crue est certes lié à l'environnement local (excellence de la terre, région assez préservée des fortes pluies de mousson et des cyclones), mais c'est surtout un choix culturel. Malgré la technique de la bauge, moins résistante que la terre damée par exemple, les habitants de Garh prouvent que leurs maisons s'inscrivent dans la durée.

D'après M. M. Ali (1985 : 153), qui cite des statistiques de 1983-84, 75 \% des maisons rurales du Bangladesh sont classées « kutcha », c'est-à-dire non permanentes et $20 \%$ ne sont pas classées; ce qui signifie, d'après l'auteur, qu'elles sont plus éphémères encore que les précédentes. Cette remarque est étonnante quand on sait que les maisons en terre peuvent durer plusieurs générations si elles sont bien entretenues et que nombre d'architectes se sont intéressés à l'architecture de terre comme moyen de construire efficacement. Des techniques nouvelles de construction en terre existent bien dans le monde, mais elles sont souvent d'un coût élevé ${ }^{24}$, donc hors de portée pour la majorité des ruraux du Bangladesh. 


\section{BIBLIOGRAPHIE}

Agarwal, Avril

1981, Mud, Mud : The Potential of Earth-based Materials for Third World Housing. Londres :

International institute for Environment and Development.

Ahmed, J.I.

1994, Up to the Waist in Mud. Earth-based Architecture in Rural Bangladesh. Dhaka : University Press Ltd.

Ali, Meer Mobbashsher

1985, « Architecture in rural areas of Bangladesh », pp. 153-155, in Exploring Architecture in Islamic Cultures 2. Regionalism in Architecture. Singapore : Concept Media Pte Ltd for the Aga Khan Award for Architecture.

Bangladesh Population Census

1991-95, Zila : Bogra. Bangladesh Bureau of Statistics.

Bernard Vincent, M.-Françoise Boussac, J.-Yves Breuil \& J.-François Salles 2001, «Excavation at the eastern Rampart Area (1993-1999). Preliminary report of the French Team », pp. 74-160, in J.-F. Salles et M. S. Alam (eds), France-Bangladesh joint venture excavations at Mabasthangarh, First interim report 1993-1999. Ville Maison de l'Orient Méditerranéen, France, Department of Archaeology, Bangladesh, Dhaka : Priyanks Printing \& Publications.

Dai, N.

1986, Report on the International Symposium on Earth Architecture. Architectural. Beijing : Institute of Japan.

Des Architectures de Terre

1981, Exposition. Paris : Centre G. Pompidou.

Hasan, Dewan Mahbub

1985, A study of traditional house forms in rural Bangladesh. Dhaka : Dept of Architecture.

Bangladesh University of Engincering \& Technology (dactylogr).

Jacqueminet, Christine, Cyril Allemand \& Kevin Pedoja

2001, «Paleoenvironment and present environment of Mahasthan, Bangladesh », pp. 19-60, in J.-

F. Salles et M. S. Alam (eds), France-Bangladesh joint venture excavations at Mahasthangarh, First interim report 1993-1999, Maison de l'Orient Méditerranéen, France, Department of Archaeology, Bangladesh, Dhaka : Priyanks Printing \& Publications,.

Lopez, O.H.

1982/83, « Construire en bambou ». Techniques et Architecture 345 : 99-101.

Mitra, A. (ed.)

1966, Census of India 1961. Vol. I, Part. IV-A. Report on House Types and Village Settlement Patterns in India. Delhi : Government of india Press.

Muktadir, Mohammed \& Dewan Mahbub Hassan

1985, « Traditional House Form in Rural Bangladesh. A case study for Regionalism in Architecture ", pp. 81-86, in Exploring Architecture in Islamic Culture 2. Regionalism in Architecture. Singapore : Concept Media Pte Ltd for the Aga Khan Award for Architecture. 
Newaj, A.

1989, Khonar bochon Krishi-o- Bangali Sanskriti. Dhaka : Bangladesh Agricultural Research Council.

Noble, William A.

1987, « Houses with Centered Courtyards in Kerala and Elsewhere in India », pp. 215-266, in P. Hockings (ed.), Dimensions of Social Life. Essays in Honor of D.G. Mandelbaum. Berlin/New York/

Amsterdam : Mouton de Gruyter.

\section{NOTES}

3. Ce terme «earth-based architecture ", déterminé par A. Agarwal (1981), lui semble plus approprié que celui de "earth architecture", architecture de terre, défini par N. Dai à l'International Symposium on Earth Architecture (1986).

4. Mahasthangarh est situé au nord-ouest du pays, à environ $250 \mathrm{~km}$ de Dhaka, la capitale.

5. Il s'agit d'un programme franco-bangladais. Nos missions ont été effectuées dans le cadre de la Mission archéologique française.

6. Dans le village de Mahasthan vivent trois familles hindoues et dans le bazar, deux autres familles intouchables qui assurent le nettoyage des latrines.

7. D.M. Hasan (1985: 22-27), qui a étudié l'ensemble de l'habitai rural, reconnaît 6 types d'habitat : 1) établissements nucléaires sur terrain plat élevé ; 2) constructions dispersées sur terres élevées artificiellement ; 3) habitat linéaire le long des fleuves ; 4) dispersé et isolé sur les îles ; 5) très concentré sur collines artificielles; 6) constructions éparpillées dans des zones de collines.

8. Il existe bien un cimetière, mais il ne sert qu'aux habitants d'un seul quartier, celui de mazar para (quartier du sanctuaire), et aux étrangers qui meurent sur place. Les habitants des autres quartiers préfèrent garder leurs morts auprès des membres de leur famille.

9. Seules deux larges mares appartiennent en commun aux familles de plusieurs para.

10. Mon-e jodi loi kosor. Pokri pabar-e tuli-o ghar (Newaj 1989 : 193). Connus sous le nom de bochon, les proverbes bengalis sont attribués à Khona qui aurait été une princesse et astrologue au $\mathrm{VI}^{\mathrm{e}}$ siècle à la cour du roi Vikramaditya. Nous remercions Shahzad Rokon Firuz pour sa traduction, et pour nous avoir fait connaître ces proverbes.

11. La résidence est patrilocale.

12. D'après les «Khonar bochon", les proverbes de Khona, il est dit: "En ce qui concerne l'hygiène, les maisons faisant face au sud sont les meilleures » (Dhokinn duari gharer raja pub duari tari proja. Poshchim duarir mukhe chai uttar duarir khajna nai), (Newaj 1989 : 196). D.M. Hasan (1985 : 53) relève que les ruraux préfèrent orienter leurs maisons vers le sud, mais, en fait, toutes les directions sont adoptées.

13. Les vaches sont rentrées à l'étable pour la nuit car les villageois craignent les vols. Pendant la journée, les animaux sont laissés à l'extérieur de l'unité d'habitation et les femmes de la maison vont les promener pour les faire pâturer.

14. D’après D.M. Hasan (1985 : 38), afin de respecter l'intimité domestique, les espaces féminin et masculin de la maison sont séparés. Ce n'est pas le cas à Garh.

15. Cette notion de bhinnu est très importante. ans ses statistiques, le gouvernement recense le nombre des foyers de chaque village.

16. Un fils quittant l'unité d'habitation de ses parents ou de ses frères et qui veut faire construire, peut démolir sa cellule et emporter la terre, le toit, les portes et fenêtres qui serviront pour sa nouvelle habitation.

17. Selon la loi de 1968 (Act $n^{\circ}$ XIV) sur les antiquités, amendée en 1976 (ordinance $n^{\circ}$ LXXIII), il est interdit «d'aliéner, détruire, déplacer... les antiquités ou de construire sur ou près d'un site 
antique ». Le directeur du musée de Mahasthangarh, chargé de faire respecter cette loi, se montre cependant tolérant. Car, outre le fait de laisser y résider les habitants qui demeurent à Garh depuis des générations, il leur permet de creuser le sol pour prélever de la terre, mais pas à plus de $30 \mathrm{~cm}$; l'utilisation du matériel archéologique, comme les briques, restant interdite (entretien personnel). Les habitants ne peuvent se plier à cette norme -à moins de faire venir de la terre de l'extérieur de Garh, ce qui serait un coût supplémentaire-, car la quantité de terre serait insuffisante s'ils ne creusaient que dans leur seule cour.

18. Il existe trois sortes de bambou (bas): le bhalko, le meilleur pour la construction car il est plein; le djawa et le tolla, qui sont creux. Pour les linteaux, c'est le bhalko ou le tolla qui sont employés (en fonction des finances du propriétaire), alors que pour la charpente et le toit c'est le bhalko. Mais chaque type de bambou prend une appellation différente selon son âge Ainsi, celui qui est utilisé pour la charpente, nommé pakha, a-t-il deux à trois ans.

19. Les habitants disent que pour construire à plus de deux niveaux, il faudrait avoir recours à des artisans professionnels. Peut-être s'agit-il plutôt d'un modèle culturel car aucun des grands propriétaires n'ont fait ce choix, ils préfèrent augmenter leur surface de terrain à bâtir et le nombre de cellules d'habitation.

20. Les plus grands propriétaires possèdent entre 15 et 25 bigha, 1 bigha équivaut à 13 ares.

21. Pour éviter que la terre ne tombe sur ses habitants à l'intérieur de la demeure, ceux-ci placent un velum en tissus sous le plafond.

22. Malheureusement, nous n'avons pas examiné comment la ferme était fixée au corps du bâtiment. D'après un architecte bangladais, les quatre coins de la ferme sont reliés par du fil de fer qui est fiché clans le sol. Nous n'avons pas observé ce procédé, mais le fil de fer était peut-être caché par un mortier. Hasan (1985 : 75) note que l'entrait de la ferme est ancré dans la terre des murs qui sont sous le plafond. D'après les habitants, rien n'est prévu pour consolider la toiture qui risque d'être emportée par un cyclone.

23. 1 euro $=60$ taka (tk). Le salaire d'un journalier varie de 50 à 80 tk par jour pour les travaux agricoles. Un kg de riz décortiqué de type IRRI de $2^{\text {ème }}$ catégorie valait 17 tk en 2001.

24. Voir à ce propos K..I. Ahmed (1994:60-86) et « Des Architectures de terre » (1981).

\section{RÉSUMÉS}

La particularité des maisons rurales de Mahasthangarh au Bangladesh est qu'elles sont construites en terre archéologique. Il s'agit de maisons à cour, un modèle assez répandu dans toute l'Asie du sud. La cour constitue la partie centrale de l'unité d'habitation dans laquelle sont effectuées nombre d'activités. La description de l'unité d'habitation comprend les différents espaces occupés par les membres de la famille, les techniques de construction dont les acteurs sont tout autant les hommes, les femmes et les enfants, mais où l'entraide entre voisins et parents n'existe pas.

The characteristic feature of rural houses in Mahasthangarh (Bangladesh) is that they are made with archaeological mud-mud from archaeological sites. These houses have associated yards, a quite widespread pattern in South Asia. The yard is the main part of the habitation, where many activities ate carried out. These activities and the different living places of the family are described. Men and women along with children participate to the construction of the house but mutual aid between neighbours and relatives does not exist. 
La peculiaridad de las casas rurales de Mahasthangarh, en Bangladesh, consiste en su construcción a base de «tierra arqueológica ». Se trata de casas con patios - un modelo bastante corriente en el conjunto de Asia meridional. El patio es la parte central del edificio, en la cual se practican numerosas actividades, y cuya descripción incluye los distintos espacios ocupados por cada miembro de la familia, las técnicas de construcción y sus actores (hombres, mujeres, niños) - sin ayuda mutua entre parientes o vecinos.

\section{INDEX}

Mots-clés : architecture, Bangladesh, construction, habitation, terre crue

\section{AUTEURS}

\section{ANIE MONTIGNY}

Musée de l'Homme, UMR 8098 « Techniques et Culture »

\section{SÉVERINE SANZ}

archéologue, Maison de l'Orient et de la Méditerranée, FR 538 